\title{
ARTICLE
}

\section{Tackling the NHS glaucoma clinic backlog issue}

\author{
David Charles Broadway ${ }^{1,2} \cdot$ Karen Tibbenham ${ }^{1}$
}

Received: 2 December 2018 / Revised: 21 February 2019 / Accepted: 30 April 2019 / Published online: 20 May 2019

(c) The Royal College of Ophthalmologists 2019

\begin{abstract}
Background/Objectives to determine whether mass case review, carried out by glaucoma sub-specialist consultants, for patients for whom there was insufficient clinic capacity, could aid reduction of the glaucoma clinic appointment backlog. Subjects/Methods patient hospital notes were reviewed by a glaucoma fellowship trained consultant and a decision was made as to whether the planned review was appropriate. Decisions were made with respect to timing, clinic-type and necessity for follow-up, together with an assessment as to whether visual field testing was required.

Results in a 3-year study a total of 9290 cases were included in the study. After consultant review, 5521 (59.5\%) patients were kept within the hospital eye service (HES) and an additional 1350 (14.5\%) had their next appointment delayed, 384 (4\%) were discharged to specialist community glaucoma optometrists and $2035(22 \%)$ were discharged to their standard community optometrists. Overall, therefore $26 \%$ of patients were discharged from the HES. Of the planned 9290 appointments, simultaneous visual field testing had been planned for 5393 patients (58\%), but after consultant review only $65 \%(n=3482)$ of these were considered necessary, reducing the number of required visual field tests by $35 \%(n=1911)$. Conclusions the authors suggest that ophthalmology departments experiencing significant clinic appointment backlog issues, consider utilising trained glaucoma sub-specialist consultants to review planned follow-up management of patients within a backlog deficit.
\end{abstract}

\section{Introduction}

Glaucoma or suspicions of glaucoma affect a large number of patients, many requiring treatment and the majority requiring regular monitoring. Glaucoma is an age-related condition and with a growing elderly population, together with more rigorous community optometric screening programme, has both a rising prevalence and incidence in the UK, as well as throughout the world [1]. Furthermore, together with glaucoma, other ophthalmic conditions [2] including diabetic eye disease [3], cataract [4] and agerelated macular degeneration (ARMD) [5] have, in recent years, increased the workload requirements of the National Health Service (NHS) hospital eye service (HES) [6]. The

David Charles Broadway

davidbroadway@waitrose.com

1 Directorate of Ophthalmology, Norfolk \& Norwich University Hospital NHS Foundation Trust Colney Lane, Norwich NR4 7UY, UK

2 School of Pharmacy, University of East Anglia, Norwich Research Park, Norwich NR45 7TJ, UK service delivery problem for ophthalmic care has been compounded by the introduction of new therapies and increased expectations for improved outcomes, especially for wet ARMD and the frequently required intra-vitreal injections of anti-vascular endothelial growth factor (antiVEGF) drugs. Management of acute disease and conditions with risk of acute visual deterioration clearly has to take priority. However, the downside of this approach is that when there is limited clinic capacity there is potential for backlogs to build up for patients with more chronic, slowly progressive conditions, as is the case with most types of glaucoma. Many UK ophthalmology departments, therefore, currently have insufficient clinic capacity to see all their 'glaucoma' follow-up patients.

The National Patient Safety Agency (NPSA) issued a rapid response report in 2009 having highlighted glaucoma patients as being particularly susceptible to harm following delayed follow-up [7]. In the NPSA report 44 patients with glaucoma were identified to have deteriorated, 13 experiencing total loss of vision during a 4 year period [7]. Furthermore, in an analysis of a large number of Moorfields Eye Hospital ophthalmic patient episodes lost to follow-up, 16 serious incidents were identified, of which 14 patients had glaucoma and another a medical retina condition 
associated with secondary glaucoma, emphasising the importance of providing appropriate follow-up intervals in a glaucoma service [8].

In 2012 the mean difference between intended and actual monitoring interval for the Moorfields Eye Hospital glaucoma service was $5.6( \pm 2.9)$ months, representing a 42.6 $( \pm 14.5) \%$ increase in interval [9]. At the Norfolk \& Norwich University Hospital (NNUH) NHS Foundation Trust the clinic administration staff estimated that in 2013 there was an approximate 3000 patient appointment backlog for glaucoma clinics with an average delay of approximately 6 months.

The NPSA asked NHS secondary care organisations to review their appointment and service provision systems to minimise the risk of irreversible and avoidable sight loss for patients with manifest or suspected glaucoma; this advice initiated the present project [7].

The aim of the present study was to analyse glaucoma appointment backlog data with a view to determining whether all appointments were appropriate and ensure that no patient was considered to be an unacceptable risk in having their appointment delayed, as suggested by the 2009 NSPA report [7].

\section{Materials (subjects) and methods}

The study was approved by the institutional audit department and followed the tenants of the Declaration of Helsinki. For a 3-year period of planned outpatient department glaucoma clinic appointments (backlog patients), a glaucoma fellowship trained consultant reviewed patient hospital notes and a decision was made as to whether the planned review was appropriate. No specific protocol was utilised but the adjusted decisions were planned to meet criteria suggested by glaucoma NICE guidelines, specific criteria being appropriate for $80 \%$ of patients $80 \%$ of the time. In order to ensure that any individual clinician bias was accounted for, a proportion of the notes review was carried out by two other glaucoma fellowship trained consultants and the outcomes compared. The reviewing consultant reviewed approximately $40-50$ patient notes in a standard NHS $4 \mathrm{~h}$ session. The notes were obtained and tracked from the medical records department by Band 2 administration assistants, as opposed to medical secretaries, who ordered them in batches of about 250 , making it an efficient process. A series of pro forma letters were used by the consultant to inform the patient and their General Practitioner of the outcomes; this process minimising additional secretarial and administration costs.

For each patient appointment the reviewing consultant categorised the appointment as either appropriate or inappropriate (too late or too soon) and the requirement for a visual field test at the planned appointment was reviewed and altered if deemed appropriate. The magnitude of any suggested expedition or further delay to any appointment was recorded. Follow-up glaucoma options available for the NNUH glaucoma service were defined as either within a consultant-led glaucoma clinic or a specialist nurse glaucoma clinic. Options for patients discharged from the HES were to either a specialist community glaucoma optometrist or a standard community optometrist, dependent on the perceived risk of future development of glaucoma for glaucoma suspects. The patients discharged to a standard community optometrist were considered to be at low risk of developing glaucoma, having been stable low-risk glaucoma suspects for at least 5 years, or to have normal eyes, with minimal risk of developing glaucoma. The patients discharged to a specialist community optometrist were lowrisk glaucoma suspects with less than 5 years of documented stabilty (see Table 1 and Fig. 1).

Data for each patient was recorded in an MS Excel spreadsheet, as well as on the NNUH patient database. The MS Excel spreadsheet data was analysed for data collected from January 2013 to December 2015. Statistical analyses were undertaken using SPSS v21.0 (SPSS, Chicago, IL, USA). Chi-squared tests were used to compare the management decisions made by the different consultants, utilising a $p$-value of $<0.05$ to indicate statistical significance.

\section{Results}

The 3-year study was carried out from January 2013 to December 2015. A total of 9301 patient notes were reviewed. After excluding 11 patients who had died, a remaining 9290 cases were included in the study. The primary researcher (DCB) reviewed $5683(61 \%)$ of the cases and two other consultants reviewed an additional 3607 (39\%) cases. There was no significant difference between the results on the basis of which consultant carried out the review and the presented results relate to the whole cohort.

The 9290 backlog patient appointments had an initially planned interval from their last appointment ranging from 2-24 months (mean $=11, \mathrm{SD}=5$ ). The planned appointments were for consultant-led clinics $(n=6014 ; 65 \%)$ and nurse-led virtual monitoring clinics $(n=3276 ; 35 \%)$, both within the hospital eye service (HES). The major initially planned use of the nurse-led HES clinic was for annual reviews (47\%) and the consultant-led HES clinics for the 2-4 month (84\%) and 18-24 month (78\%) follow-up appointments (see Table 2).

After consultant review, although 5521 (59.5\%) patients were kept within the HES with minimal suggested change to their planned appointment, an additional 1350 (14.5\%) had their next appointment delayed, 384 (4\%) were 
Table 1 Potential decisions

\begin{tabular}{|c|c|c|c|}
\hline & Urgency & Visual fields & Clinic \\
\hline 1 & See urgently & \pm fields & Consultant HES clinic \\
\hline 2 & See as soon as possible & \pm fields & $\begin{array}{l}\text { 2a HES clinic as planned } \\
\text { 2b 'downgraded' HES clinic } \\
\text { 2c 'upgraded' HES clinic }\end{array}$ \\
\hline 3 & $\begin{array}{l}\text { See with additional delay of } \\
\text { ' } x \text { ' months }\end{array}$ & \pm fields & $\begin{array}{l}\text { 3a HES clinic as planned } \\
\text { 3b 'downgraded' HES clinic } \\
\text { 3c 'upgraded' HES clinic }\end{array}$ \\
\hline 4 & Discharge & Optometrist to decide & $\begin{array}{l}\text { 4a Specialist community glaucoma } \\
\text { optometrist }\end{array}$ \\
\hline
\end{tabular}

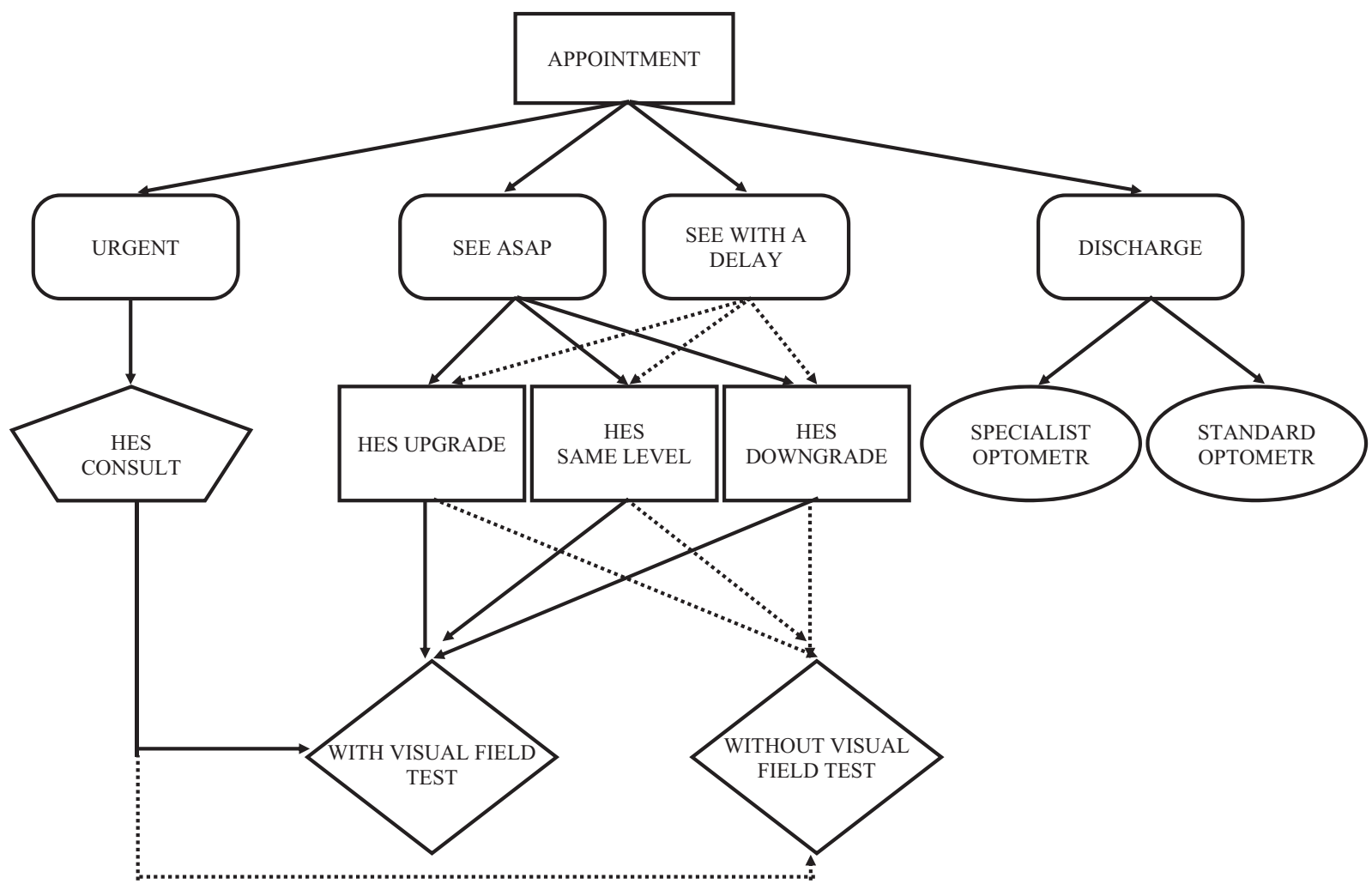

Fig. 1 A flow-chart showing the decision making pathways in potentially changing the planned follow-up appointment to a more appropriate appointment (ASAP as soon as possible, HES hospital eye service)

discharged to specialist community glaucoma optometrists (as low-risk glaucoma suspects, not proven to be stable for 5 years) and 2035 (22\%) were discharged to their standard community optometrists (as either normal, or as low-risk glaucoma suspects, proven to be stable for 5 years). Overall, therefore $26 \%$ of patients were discharged from the HES (Fig. 2). With respect to the initially planned interval between appointments, the discharge rate increased significantly with increasing interval duration, being only $6 \%$ for those planned for 2-4 month appointments and rising to
$61 \%$ for those planned for $18-24$ month appointments (see Table 2).

The majority ( $n=5497 ; 59 \%)$ of HES clinic appointments were kept at the same level, with only a small number ( $n=195 ; 2 \%$ ) being upgraded (with only 6 of these being upgraded to urgent). Of the 6 patients seen urgently, 2 were found to have progressive disease and their management was altered, whereas 4 were considered to be stable. A significant minority $(n=3598 ; 39 \%)$ of initially planned appointments were downgraded; 1312 (36.5\%) by one 
Table 2 The initial planned appointments and how they were adjusted after consultant review

\begin{tabular}{|c|c|c|c|c|c|}
\hline & \multicolumn{4}{|c|}{ Initial planned appointment interval (mean \pm standard deviation) } \\
\hline & & $2-4$ months $(3 \pm 1)$ & $6-9$ months $(7 \pm 1)$ & 12 months $(12 \pm 0)$ & $18-24$ months $(21 \pm 3)$ \\
\hline \multirow[t]{4}{*}{ Initial planned appointment } & $n$ & 456 & 3572 & 4015 & 1247 \\
\hline & HES Cons & $84 \%(n=381)$ & $71 \%(n=2541)$ & $53 \%(n=2123)$ & $78 \%(n=967)$ \\
\hline & HES Nurse & $16 \%(n=75)$ & $29 \%(n=1031)$ & $47 \%(n=1892)$ & $22 \%(n=280)$ \\
\hline & $+\mathrm{VF}$ & $37 \%(n=170)$ & $48 \%(n=1707)$ & $31 \%(n=1234)$ & $78 \%(n=968)$ \\
\hline \multirow{13}{*}{$\begin{array}{l}\text { Suggested appointment } \\
\text { after consultant review }\end{array}$} & Kept in HES & $77 \%(n=350)$ & $56 \%(n=1990)$ & $67 \%(n=2701)$ & $38 \%(n=480)$ \\
\hline & $\begin{array}{l}\text { HES } \\
\text { with delay }\end{array}$ & $17 \%(n=77)$ & $31 \%(n=1122)$ & $4 \%(n=139)$ & $1 \%(n=12)$ \\
\hline & Discharge & $6 \%(n=29)$ & $13 \%(n=460)$ & $29 \%(n=1175)$ & $61 \%(n=755)$ \\
\hline & Discharge SO & $0.2 \%(n=1)$ & $1.6 \%(n=53)$ & $4 \%(n=173)$ & $13 \%(n=157)$ \\
\hline & Discharge $\mathrm{OO}$ & $5.8 \%(n=28)$ & $11.4 \%(n=407)$ & $25 \%(n=1002)$ & $48 \%(n=598)$ \\
\hline & Upgrade & $1 \%(n=4 ; 0$ urgent $)$ & $2 \%(n=65 ; 4$ urgent $)$ & $2 \%(n=98 ; 1$ urgent $)$ & $2 \%(n=28 ; 1$ urgent $)$ \\
\hline & No change & $73 \%(n=333)$ & $67 \%(n=2401)$ & $59 \%(n=2367)$ & $32 \%(n=396)$ \\
\hline & Downgrade & $26 \%(n=119)$ & $31 \%(n=1106)$ & $39 \%(n=1550)$ & $66 \%(n=823)$ \\
\hline & -1 level & $20 \%(n=91)$ & $19 \%(n=661)$ & $12 \%(n=463)$ & $8 \%(n=97)$ \\
\hline & -2 level & $1 \%(n=5)$ & $3 \%(n=119)$ & $10 \%(n=393)$ & $19 \%(n=243)$ \\
\hline & -3 level & $5 \%(n=23)$ & $9 \%(n=326)$ & $17 \%(n=694)$ & $39 \%(n=483)$ \\
\hline & $+\mathrm{VF}$ & $36 \%(n=154)$ & $44 \%(n=1355)$ & $30 \%(n=856)$ & $72 \%(n=354)$ \\
\hline & VF reduction & $9 \%(n=16)$ & $21 \%(n=352)$ & $31 \%(n=378)$ & $63 \%(n=614)$ \\
\hline
\end{tabular}

$n$ number, HES Hospital Eye Service, $V F$ Visual Field, SO Specialist [glaucoma] Optometrist, $O O$ Own [standard] Optometrist

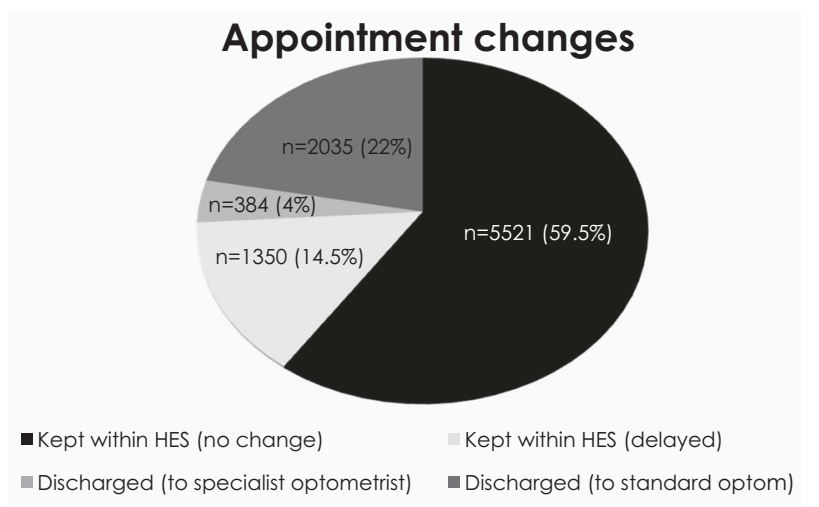

Fig. 2 A pie-chart illustrating that although it was suggested that the majority of patients kept their original follow-up appointment $(59.5 \%)$, after review of the hospital notes, a significant proportion $(26 \%)$ were discharged from the HES (mainly to a standard community optometrist) or had their HES appointment delayed (14.5\%)

level, $760(21 \%)$ by two levels and 1526 (42.5\%) by three levels.

For the initially planned 9290 appointments, simultaneous visual field testing had been planned for 5393 patients $(58 \%)$, but after consultant review only $65 \%(n=$ 3482 ) of these were considered necessary, reducing the number of required visual field tests by $35 \%(n=1911)$ (Fig. 3). With respect to the initially planned interval between appointments, the rate of visual field test cancellation increased significantly with increasing interval duration, being only $9 \%$ for those planned for 2-4 month appointments and rising to $63 \%$ for those planned for 18-24 month appointments (see Table 2). The field tests deemed unnecessary included those booked for patients that were discharged to have follow-up with a community optometrist.

\section{Discussion}

Patients with glaucoma should be monitored according to risk of progressive visual loss. Monitoring interval guidance has been outlined by organisations including the National Institute of Clinical Excellence (NICE) [10], the American Academy of Ophthalmology (AAO) [11] and the European Glaucoma Society (EGS) [12]. Delay in glaucoma patient follow-up has been reported as a significant cause of progressive visual field loss, including total loss of vision, that might have been preventable with appropriate follow-up and altered management [7,9].

The ability to identify and analyse glaucoma backlog data in Norwich resulted from an operational need to reduce the number of ophthalmology clinic appointments altered from one day to another. Historically, appointments were booked up to 18 months in advance and staff changes and leave bookings resulted in the need to move many 


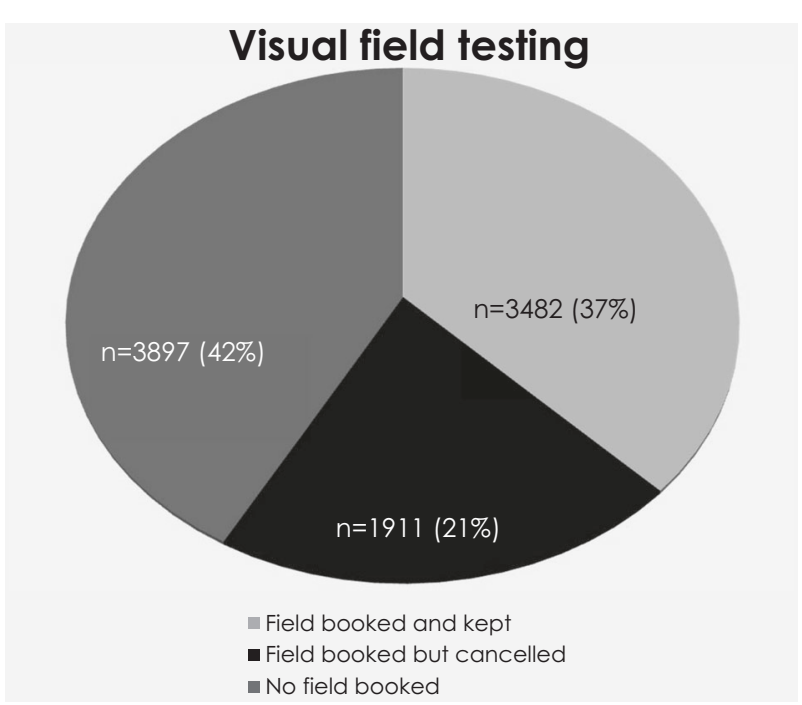

Fig. 3 A pie-chart illustrating that of the $58 \%$ of patients booked for field tests at follow-up appointments, review of the hospital notes resulted in $35 \%$ of the tests being cancelled as unnecessary

appointments, sometimes on more than one occasion. Appointment alterations resulted in patient inconvenience and extra work for administration staff. In addition, appointment changes often resulted in longer waits than originally intended for the patient. Since no target date was recorded on the NNUH patient database it was not possible to track how long over the original desired follow-up interval the patient had waited. As a result the NNUH switched to a system whereby the patient was advised in clinic how long their follow-up interval would be and they were asked to telephone six weeks prior to that to book the actual appointment. A 6 week interval was chosen to fit with the NNUH 6 week leave booking rule, such that clinic administration staff would know which clinical staff would be available. Initially all appointments were booked in this manner but later any appointments planned to be within 4 months were booked without delay. In order to ensure that patients were not lost to follow-up, each patient was entered onto an outpatient waiting list database, with a follow-up interval and clinic type, together with grade of clinical staff member that should review the patient (specialist nurse, junior doctor, consultant), specified for each entry.

Establishing a database, created the opportunity to produce reports that could track aggregate patient data and helped the department to understand the shortfall in appointment capacity versus demand. The plan was to produce hard data that could aid future commissioning of services to address the shortfall. In the shorter term the process allowed the department to put processes in place to manage the backlog of patients. Given that it was not possible to gain extra capacity immediately, a consultant with a sub-speciality interest in glaucoma embarked upon a review of the medical notes of the patients who had passed their appointment target date, starting with the longest waiters. Notes were reviewed to assess whether the original specified follow-up interval and the suggested grade of reviewing staff was appropriate and changes were made as deemed necessary.

An initial pilot study review of a few hundred patient notes indicated that there was a higher than expected number of inappropriate follow-up intervals. It was considered appropriate, therefore to instigate an additional review of the medical notes for patients who had already been booked into future clinics and made adjustments as necessary. It was considered that the review of established appointments and appropriate alterations to these would be useful in increasing short-term capacity for urgent reviews.

The results of the final study revealed that the reviewing consultants considered that a higher than expected number of patients (approximately a quarter) could be discharged from the HES glaucoma clinics (26\%), Furthermore 39\% of the follow-up appointments were reallocated to more costeffective pathways. A large number of initially planned visual field tests were cancelled, reducing the number required by $35 \%$, resulting in significant cost savings and reduced unnecessary testing of patients who often find such assessments stressful.

With respect to the planned interval between an initially planned appointment and that suggested after consultant review, a sub-group analysis of the data revealed that the longer the planned interval the greater the discrepancy between planned and suggested appointment timings (see Table 2). There was a similar effect on the reviewing consultant's opinion as to the need for a visual field test, this reducing by $63 \%$ for those initially planned to have 18-24 month follow-up.

Tatham and Murdoch [9] reported that the Moorfields Eye Hospital glaucoma clinical staff were selecting appropriate monitoring intervals for patients and that it was hospitalinitiated appointment rescheduling that was the problem. However, their study was for patients with POAG alone and did not include patients with other types of glaucoma, suspicions of glaucoma or ocular hypertension. In the present study all patients within the NNUH glaucoma service were included and it appeared that there was a tendency for clinical staff to be too risk averse with respect to low-risk glaucoma suspects, or those with mild ocular hypertension, whom a sub-speciality trained glaucoma consultant considered appropriate for discharge out of the HES. Whereas there is a well-established definition of manifest POAG and guidance for the monitoring and management of POAG, the definition of a glaucoma suspect is less specific and open to interpretation of clinical signs, something which has the potential to be more difficult for junior clinical staff who are frequently allocated to work in glaucoma clinics or see patients with glaucoma in general clinics. 
Patient safety should be of prime concern with respect to healthcare and quality of care has fortunately become under increased scrutiny and linked to financial reimbursement schemes [13] measured against the standards of organisations such as NICE, the AAO, the EGS and the Royal College of Ophthalmologists (RCOphth). Recommended RCOphth quality indicators for glaucoma services have included having named clinical leads for glaucoma, using dedicated glaucoma review clinics, auditing outcomes and the maintenance of review intervals to within $15 \%$ of those intended [14]. In the current study, patient safety could be questioned with a high discharge rate and a significant recommended delay to appointments. However, the reviewing consultants were using guidance from NICE, the AAO and the EGS to ensure that patient safety was paramount. Indeed, the discharge of patients with no evidence for glaucoma, grants greater access to those with manifest glaucoma, potentially improving safety and outcomes. Furthermore, it should be remembered that patients for whom glaucoma has been excluded are still able to have continuing glaucoma screening with their own community optometrists to ensure appropriate re-referral back to the HES, should this be deemed necessary. Strict adherence to NICE guidelines can be inappropriate for certain patients and as an example a patient that is incapable of producing reliable visual fields should not be subjected to such testing on NICE guidance alone. In fact it is stated that NICE guidance should be adhered to for at least $80 \%$ of patients, $80 \%$ of the time (i.e., for at least only $64 \%$ of events) [10]. Understandably, junior clinical staff have a tendency to be more risk averse than more experienced colleagues and the involvement of the latter has the potential to improve efficiency without reducing quality or safety. In the present study, only $2 \%$ of patients were upgraded with respect to their clinic follow-up and only 6 patients were recommended for urgent review. Of the 6 patients seen urgently 2 were found to have progressive disease and their management was altered - these patients might have deteriorated further had they not been identified during the present study.

The study had limitations. Firstly, a full economic analysis was not performed. However, by reviewing 40-50 notes/session the consultant was able to review the management of significantly more patients than would have been possible to see in an out-patient clinical session. By discharging and downgrading follow-up appointments, potentially improving the quality of care and reducing the number of visual field tests required, the long-term economic savings are almost certainly significant. Secretarial and administration costs of the process were not analysed, but the use of pro forma letters selected and prepared by the reviewing consultant minimised these costs. Secondly, the grade of clinician whose last clinical decision with respect to follow-up that was being assessed was not documented. Not surprisingly the reviewing consultant considered that he rarely altered follow-up decisions when made by either himself or another consultant with a sub-speciality interest in glaucoma, but was more likely to change those decisions made by the more junior or inexperienced clinician; however, this was not analysed formally.

In the longer term it is hoped that more objective diagnostic categorisation and methods for detecting progressive disease will become available to reduce the necessity for the current dependence on more subjective clinical skills [15]. In the shorter term, however, utilising fellowship-trained glaucoma sub-specialist consultants to improve glaucoma services has potential to improve quality and efficiency. It is acknowledged that the present report relates to a singlecentre study, although it is important to note that there was agreement between all three of the consultants who reviewed patient case notes with respect to decision making, despite the fact that agreement between clinicians relating to glaucoma-specific parameters do not always have high agreement rates [15-17]. In one key survey, Malik and coworkers identified a large variation in the attitudes of $70 \mathrm{UK}$ and Eire glaucoma specialists with respect to appropriate follow-up and visual field testing intervals, these frequently being inconsistent with NICE guidelines [16]. Although it is accepted that a research-recommended routine of six visual field examinations in an initial two year period is best for identifying progressive visual field loss [18, 19], many regard this as impractical in the current NHS heath-care setting. NICE has in fact recognised that there is currently a lack of evidence regarding the appropriate frequency of monitoring intervals (and visual field testing) for patients with glaucoma [10]. NICE have recommended that future research is carried out with respect to determining best practice and the optimum frequency for visual field testing in the management of glaucoma [10]. Another issue of importance is that relating to the utilisation of junior staff during their training in specialist clinics. Juniors often have workloads that do not permit input from a more senior clinician on an individual patient basis. Perhaps an ideal situation would be for trainees to be supernumerary until sufficiently trained. However, with backlog issues the desire to have trainees, together with non-glaucoma sub-specialists, offering a service commitment within a glaucoma service is of course high.

In conclusion, the authors suggest that ophthalmology departments within the NHS HES, experiencing significant clinic appointment backlog issues, consider utilising trained glaucoma sub-specialist consultants to review planned follow-up management of patients within a backlog deficit. In the longer term it is suggested that, where possible, glaucoma sub-specialist consultants are more involved in the planning of future follow-up appointments to minimise the development of future unnecessary clinic backlog issues. 


\section{Summary}

\section{What was known before}

- There are significant glaucoma clinic appointment backlog issues in many ophthalmology departments

- For patients with glaucoma, delay in follow-up can result in significant progressive deterioration

- Progressive deterioration in glaucomatous optic neuropathy is irreversible and may lead to immediate or eventual loss of vision

- Junior clinicians are probably risk averse

\section{What this study adds}

- Evidence that utilising trained glaucoma sub-specialist consultants to review planned follow-up management of patients within a backlog deficit can significantly reduce the deficit

- Junior clinicians are frequently risk averse

- The suggestion that glaucoma sub-specialist consultants should be more involved in the planning of all future follow-up appointments to minimise the development of future unnecessary clinic backlog issues

\section{Compliance with ethical standards}

Conflict of interest The authors declare that they have no conflict of interest.

Publisher's note: Springer Nature remains neutral with regard to jurisdictional claims in published maps and institutional affiliations.

\section{References}

1. Tham YC, Li X, Wong TY, Quigley HA, Aung T, Cheng CY. Global prevalence of glaucoma and projections of glaucoma burden through 2040: a systematic review and meta-analysis. Ophthalmology. 2014;121:2081-90.

2. Klein BE, Klein R. Projected prevalences of age-related eye diseases. Inv Ophthalmol Vis Sci. 2013;54:ORSF14-17.

3. Thomas RL, Dunstan FD, Luzio SD, Chowdhury SR, North RV, Hale SL, et al. Prevalence of diabetic retinopathy within a national diabetic retinopathy screening service. $\mathrm{Br} \mathrm{J}$ Ophthalmol. 2015;99:64-8.
4. Prokofyeva E, Wegener A, Zrenner E. Cataract prevalence and prevention in Europe: a literature review. Acta Ophthalmol. 2013;91:395-405.

5. Wong WL, Su X, Li X, Cheung CM, Klein R, Cheng CY, et al. Global prevalence of age-related macular degeneration and disease burden projection for 2020 and 2040: a systematic review and meta-analysis. Lancet Glob Health. 2014;2:e106-16.

6. Chalk D, Smith M. Guidelines on glaucoma and the demand for services. Br J Healthc Manag. 2013;19:476-81.

7. National Patient Safety Agency. Preventing delay to follow-up for patients with glaucoma. Available at Rapid Response Report. 2009. http://www.nrls.npsa.nhs.uk/resources/?entryid45=61908\&p=2. Accessed 01 June 2018.

8. Davis A, Baldwin A, Hingorani M, Dwyer A, Flanagan D. A review of 145234 ophthalmic patient episodes lost to follow-up. Eye. 2017;31:422-9.

9. Tatham A, Murdoch I. The effect of appointment rescheduling on monitoring interval and patient attendance in the glaucoma outpatient clinic. Eye. 2012;26:729-33.

10. NICE (editor). CG85 Glaucoma: NICE guideline. In: Published clinical guidelines. 1st ed. National Institute of Health and Clinical Excellence. London: National Collaborating Centre for Acute Care at The Royal College of Surgeons of England; 2009.

11. American Academy of Ophthalmology Preferred Practice Pattern Panel. Primary open-angle glaucoma PPP-2015. San Francisco, CA: American Academy of Ophthalmology: Hoskins Center for Quality Eye Care; 2015.

12. European Glaucoma Society. Terminology and guidelines for glaucoma. 4th ed. Savona, Italy: European Glaucoma Society; 2014.

13. Department of Health. Using the commissioning for quality and innovation (CQUIN) payment framework. 2008. http://www.dh. gov.uk/en/Publicationsandstatistics/Publications/Publica tionsPolicyAndGuidance/DH_091443. Accessed 01 June 2018.

14. The Royal College of Ophthalmologists Quality Standard Development Group. Quality standards for glaucoma services. 2011. http://www.rcophth.ac.uk/page.asp?section $=444\{$ sectionsign $\}$ ionTitle $=$ Quality + Standards. Accessed 01 June 2018.

15. Reus NJ, Lemij HG, Garway-Heath DF, Airaksinen PJ, Anton A, Bron AM, et al. Clinical assessment of stereoscopic optic disc photographs for glaucoma: the European Optic Disc Assessment Trial. Ophthalmology. 2010;117:717-23.

16. Malik R, Baker H, Russell RA, Crabb DP. A survey of attitudes of glaucoma subspecialists in England and Wales to visual field test intervals in relation to NICE guidelines. BMJ open. 2013;3: e002067. Jan 1.

17. ECCTS Kwun, Nikita E, Lim J, Spencer F, Au L. Virtual glaucoma clinic: do consultants agree on management outcomes. J Clin Exp Ophthalmol. 2017;7:585.

18. Chauhan BC, Garway-Heath DF, Goñi FJ, Rossetti L, Bengtsson $\mathrm{B}$, Viswanathan AC, et al. Practical recommendations for measuring rates of visual field change in glaucoma. Br J Ophthalmol. 2008;92:569-73.

19. Garway-Heath DF, Crabb DP, Bunce C, Lascaratos G, Amalfitano $\mathrm{F}$, Anand $\mathrm{N}$, et al. Latanoprost for open angle glaucoma (UKGTS): a randomised, multicentre, placebo-controlled trial. Lancet. 2015;385:1295-304. 